\title{
Increased plasma lipoprotein-associated phospholipase A2 levels are associated with coronary slow flow
}

Yao-dong Ding ${ }^{1}$, Yu-qiang Pei', Rui Wang ${ }^{1}$, Jia-xing Yang ${ }^{1}$, Yin-xin Zhao ${ }^{1}$, Xiao-li Liu', Hua Shen ${ }^{1}$, Qian Ma', Shuo Zhang ${ }^{2}$ and Hai-long Ge 1* $^{*}$

\begin{abstract}
Objective: Coronary slow flow (CSF) is characterized by delayed opacification of distal epicardial coronary arteries without significant coronary stenosis. In addition, The changes of lipoprotein-associated phospholipase A2 ( $\left.L p-P L A_{2}\right)$ as a significant predictive factor for CSF remain controversial. The study aims to investigate the association between plasma LP-PLA 2 and CSF.

Methods: In this retrospective study, 170 consecutive patients who underwent coronary angiography were enrolled in Beijing Anzhen Hospital from January 2017 to September 2019, and were divided into CSF group and normal control groups. According to coronary blood flow rate measured by the thrombolysis in myocardial infarction frame count (TFC) method, CSF was defined as TFC > 27. Serum Lp-PLA 2 levels were measured in an enzyme-linked immunosorbent assay.

Results: Lp-PLA 2 levels were higher in the CSF group than in the control group (288.6 \pm 50.3 versus $141.9 \pm 49.7$, $P<0.001)$ and were significantly correlated with the mean coronary artery thrombolysis in myocardial infarction (TIMI) frame count $(r=0.790, P<0.001)$. Logistic regression analysis showed that high $L p-P L A_{2}$ was independently associated with CSF after adjustment for conventional risk factors $(O R=1.040, C l=1.022-1.059, P<0.001)$. Male sex $(\mathrm{OR}=2.192, \mathrm{Cl}=1.161-4.140, P=0.016)$ and hypertension $(\mathrm{OR}=1.965, \mathrm{Cl}=1.034-3.736, P=0.039)$ were also CSF risk factors. Receiver-operating characteristic curve (ROC) analysis showed that Lp-PLA 2 levels can predict CSF severity; the predictive power was higher than the other risk factors.

Conclusion: Our study demonstrated that patients with CSF had higher circulating levels of Lp-PLA 2 than normal controls. After adjustment for potential confounders, increased $L p-P L A_{2}$ was independently associated with presence of CSF.
\end{abstract}

Keywords: Coronary slow flow, Lipoprotein-associated phospholipase A2, Coronary artery disease

\footnotetext{
*Correspondence: gehailong@126.com

'Department of Cardiology, Beijing Anzhen Hospital, Capital Medical University, Beijing 100029, PR China

Full list of author information is available at the end of the article
}

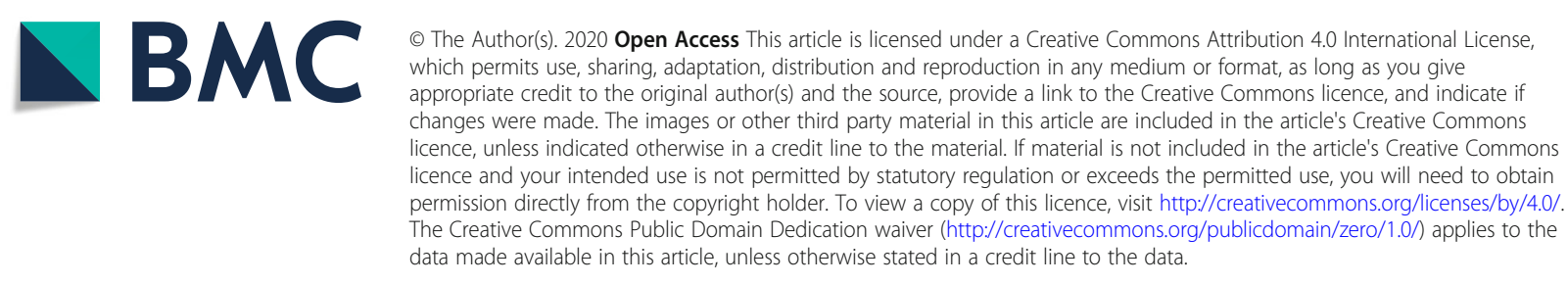


The coronary slow flow (CSF) phenomenon is a special phenomenon involving coronary microcirculation dysfunction, which is characterized by delayed distal vascular opacification without significant epicardial coronary stenosis. Studies have shown that slow blood flow is often associated with adverse cardiovascular events, including angina, myocardial infarction, malignant arrhythmias and even sudden cardiac death, suggesting a poor prognosis [1]. Although it has been 40 years since Tambe et al. first discovered slow blood flow [2], the exact mechanism of its pathogenesis remains unclear. A large number of studies have shown that the pathogenesis of CSF may involve vascular endothelial dysfunction, vascular inflammation, obesity, atherosclerosis and other factors [3-5]. However, there is a lack of effective biomarkers that specifically predict CSF. Recently, a vast majority of evidence has indicated that many inflammatory mediators such as interleukin-1, interleukin-10, and the lymphocyte to monocyte ratio, are associated with the pathogenesis of CSF, indicating the presence of a proinflammatory process occurring as a result of the phenomenon [6-8]. Thus, it is of value to identify the risk factors for CSF in order to detect and prevent CSF as early as possible.

Lipoprotein-associated phospholipase A2 (Lp-PLA $A_{2}$ ), a leukocyte-derived enzyme, is involved in the metabolism of low-density lipoprotein (LDL), propagating atherogenesis, and mediating the inflammatory process of the vascular wall [9]. Lp-PLA 2 circulates in plasma in its active form with complexes of LDL and high density lipoprotein (HDL) [10]. Recent evidence suggests that Lp-PLA 2 plays an important role in the pathophysiology of atherosclerosis and as a predictive biomarker for predicting future cardiovascular events [11]. According to its properties of proinflammation and atherogenesis, and the fact that increased Lp-PLA 2 has been found to be closely associated with inflammation and atherosclerosis, we speculate that Lp-PLA $A_{2}$ may be associated with CSF [12]. Therefore, in this study, our aim was to evaluate the relationship between coronary blood flow and the Lp-PLA2 level.

\section{Methods}

\section{Study patients}

The retrospective study was carried out from January 2017 to September 2019 at Anzhen Hospital. A total of 170 patients who underwent coronary angiography with clinical chest pain and without significant coronary stenosis were consecutively enrolled in the observational study; 78 of the patients had slow coronary flow without any stenosis evidenced by coronary angiography, and 92 patients had normal coronary arteries and normal flow. Patients with prior evidence of coronary artery disease (acute coronary syndrome, coronary interventions history, coronary plaque and significant atherosclerotic lesions (stenosis above 40\%), coronary ectasia, coronary calcification), congenital heart disease, valvular heart disease, cardiomyopathy, ischemic electrocardiogram, hematological system disease, tumors, heart failure, liver dysfunction, or kidney dysfunction or who had recently undergone surgery were excluded from the study. The study was approved by the Beijing An zhen Hospital Ethics Committee of Capital Medical University, and all patients provided informed consent.

\section{Anthropometric and laboratory measurements}

The study measured demographic characteristics, including age, sex, height, weight, body mass index (BMI) and waist circumference. Smoking, hypertension, diabetes mellitus and medication use were collected from electronic medical records. After fasting for at least $8 \mathrm{~h}$, peripheral blood was collected on the morning of the first day of admission and stored at $-70{ }^{\circ} \mathrm{C}$. The plasma Lp-PLA $A_{2}$ activity was measured using an enzyme-linked immunoassay (PLAC ${ }^{\text {max }}$ test, DIADEXUS, USA). Glucose, urea, creatinine, total cholesterol (TC), triglyceride (TG), HDL, and LDL levels were measured using a chemiluminescence method with a Roche Diagnostics Cobas analyzer Cobas 8000, c702 module. High sensitivity Creactive protein (hs-CRP) levels were also measured by the nephelometric method. All samples were tested in triplicate according to the manufacturer's protocols.

\section{Diagnostic criteria}

ACS was diagnosed according to European Society of Cardiology guidelines in 2015 [13]. Cardiomyopathy was diagnosed by established histological, immunological, and immunohistochemical criteria according to the WHO classification [14]. The diagnostic criteria of diabetes mellitus was according to WHO guidelines or by indication for insulin or anti-diabetic medications [15]. Hypertension was defined as repeated systolic pressure $\geq$ $140 \mathrm{mmHg}$ and/or diastolic pressure $\geq 90 \mathrm{mmHg}$ at least twice, or previously diagnosed hypertension [16].

\section{Coronary angiography protocols}

CSF was defined according to the thrombolysis in myocardial infarction (TIMI) frame count (TFC) method. Coronary angiogram was performed using a digital subtraction angiography system (Allura Xper FD20; Philips Medical Systems, Best, the Netherlands), which used the standard Judkins technique to obtain images at the rate of $30 \mathrm{frames} / \mathrm{s}$. The injection rate was $4-5 \mathrm{ml} / \mathrm{s}$ in the left coronary artery and $3-4 \mathrm{ml} / \mathrm{s}$ in the right coronary artery (RCA). The left coronary artery was injected with 8-10 $\mathrm{ml}$ contrast agent and RCA was injected with 6-8 $\mathrm{ml}$ contrast agent. The left anterior descending coronary artery (LAD) was imaged as the right anterior oblique projection with an angle of $20-25^{\circ}$, the left circumflex coronary artery (LCX) was imaged as the right anterior 
Table 1 Clinical characteristics of the study populations

\begin{tabular}{|c|c|c|c|c|}
\hline Characteristics & Total (170) & NCF (92) & CSF (78) & $P$ value \\
\hline \multicolumn{5}{|l|}{ Clinical Characteristics } \\
\hline Age (years) & $61.6 \pm 9.7$ & $62.7 \pm 9.5$ & $60.2 \pm 9.7$ & 0.085 \\
\hline Sex(Male) & $103(60.6 \%)$ & $48(52.1 \%)$ & $55(70.5 \%)$ & 0.018 \\
\hline $\mathrm{BMI}\left(\mathrm{kg} / \mathrm{m}^{2}\right)$ & $25.3 \pm 2.8$ & $25.1 \pm 2.9$ & $25.5 \pm 2.7$ & 0.341 \\
\hline Waistline (cm) & $88.8 \pm 10.2$ & $87.9 \pm 9.5$ & $89.8 \pm 10.9$ & 0.215 \\
\hline Diabetes mellitus, n (\%) & $37(21.8 \%)$ & 19(20.7\%) & 18(23.1\%) & 0.713 \\
\hline Hypertension, n (\%) & $112(65.9 \%)$ & $67(72.8 \%)$ & $45(57.7 \%)$ & 0.051 \\
\hline Smoking, n (\%) & $23(13.5 \%)$ & $13(14.1 \%)$ & $10(12.8 \%)$ & 0.826 \\
\hline \multicolumn{5}{|l|}{ Laboratory parameters } \\
\hline FBG (mmol/L) & $6.28 \pm 1.89$ & $6.16 \pm 1.82$ & $6.41 \pm 1.98$ & 0.407 \\
\hline $\mathrm{HbA1C}(\%)$ & $2.95 \pm 3.41$ & $3.21 \pm 3.43$ & $2.65 \pm 3.38$ & 0.290 \\
\hline $\mathrm{SBP}(\mathrm{mmHg})$ & $129 \pm 15$ & $128 \pm 15$ & $129 \pm 15$ & 0.688 \\
\hline $\mathrm{DBP}(\mathrm{mmHg})$ & $76 \pm 10$ & $78 \pm 10$ & $76 \pm 10$ & 0.677 \\
\hline TG $(\mathrm{mmol} / \mathrm{L})$ & $1.72 \pm 1.19$ & $1.51 \pm 0.66$ & $1.97 \pm 1.57$ & 0.020 \\
\hline TC $(\mathrm{mmol} / \mathrm{L})$ & $4.56 \pm 0.96$ & $4.76 \pm 1.01$ & $4.32 \pm 0.85$ & 0.003 \\
\hline $\mathrm{HDL}-\mathrm{C}(\mathrm{mmol} / \mathrm{L})$ & $1.33 \pm 0.28$ & $1.49 \pm 0.29$ & $1.15 \pm 0.07$ & $<0.001$ \\
\hline LDL-C (mmol/L) & $2.63 \pm 0.77$ & $2.73 \pm 0.80$ & $2.51 \pm 0.71$ & 0.064 \\
\hline Creatinine $(\mu \mathrm{mol} / \mathrm{L})$ & $75.6 \pm 24.1$ & $75.3 \pm 24.0$ & $75.8 \pm 24.2$ & 0.870 \\
\hline $\mathrm{Hs}-\mathrm{CRP}(\mathrm{mg} / \mathrm{L})$ & $2.44 \pm 3.16$ & $2.09 \pm 3.00$ & $2.84 \pm 3.31$ & 0.127 \\
\hline WBC $\left(\times 10^{9} / \mathrm{L}\right)$ & $6.24 \pm 1.63$ & $6.12 \pm 1.69$ & $6.38 \pm 1.56$ & 0.287 \\
\hline Ejection fraction (\%) & $65.4 \pm 6.4$ & $66.1 \pm 6.3$ & $64.5 \pm 6.6$ & 0.103 \\
\hline Lp-PLA2 (ng/mL) & $209.2 \pm 88.6$ & $141.9 \pm 49.7$ & $288.6 \pm 50.3$ & $<0.001$ \\
\hline \multicolumn{5}{|l|}{ Medication } \\
\hline Beta-blockers, n (\%) & $51(70 \%)$ & 29(31.5\%) & $22(28.2 \%)$ & 0.737 \\
\hline ACEI/ARB, n (\%) & $52(30.1 \%)$ & $29(31.2 \%)$ & $23(29.5 \%)$ & 0.868 \\
\hline Statins, n (\%) & $66(38.8 \%)$ & $33(35.9 \%)$ & $33(42.8 \%)$ & 0.432 \\
\hline Insulin, n (\%) & $62(36.5 \%)$ & $36(39.1 \%)$ & $26(33.3 \%)$ & 0.523 \\
\hline
\end{tabular}

$B M I$ Body mass index, FBG Fasting blood glucose, HbA1c Glycosylated hemoglobin A1c, SBP Systolic blood pressure, DBP Diastolic blood pressure, LDL-c Low density lipoprotein cholesterol, $H D L-c$ High density lipoprotein cholesterol, $T C$ total cholesterol, $T G$ triglyceride, $H s$ - $C R P$ hyper-sensitive $C$-reactive protein, WBC white blood cell

oblique projection with an angle of $20-25^{\circ}$, and the RCA was imaged as the positive projection with an angle of $30^{\circ}$. The first frame was defined when the contrast agent touched two medial walls of the coronary artery and advanced steadily with a diameter of more than $70 \%$, and the last frame was defined when the leading edge of the contrast agent reached the end of the branches of the coronary artery. The frame counts in the LAD were divided by a factor of 1.7 to correct for its longer length. According to Gibson et al., any frame count over 27 is considered abnormal and indicates significant CSF [17]. All patients were carefully monitored for pulse and blood pressure during coronary angiogram.

\section{Statistical analysis}

Data were analyzed with IBM SPSS software version 23.0. Categorical variables were presented as frequencies

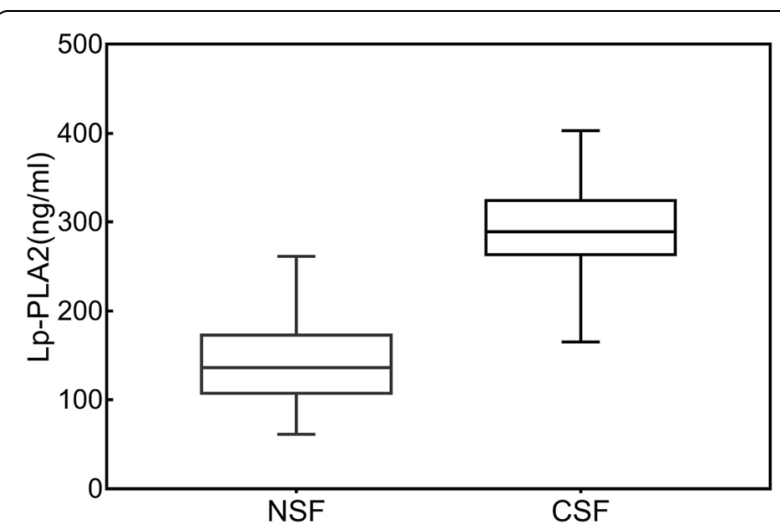

Fig. $1 \mathrm{Lp}-\mathrm{PLA} \mathrm{A}_{2}$ expressed in the CSF group compared with the patients in the NCF, the difference was statistically significant. Lp$\mathrm{PLA}_{2}$ lipoprotein-associated phospholipase A2, CSF coronary slow flow, NSF normal slow flow 
Table 2 Comparison of TIMI frame counts between patient groups with and without coronary slow flow phenomenon

\begin{tabular}{llll}
\hline Vessel & NCF $(n=92)$ & CSF $(n=78)$ & $P$ value \\
\hline LAD & $21 \pm 3$ & $31 \pm 4$ & $<0.001$ \\
LCX & $20 \pm 3$ & $30 \pm 3$ & $<0.001$ \\
RCA & $20 \pm 2$ & $32 \pm 3$ & $<0.001$ \\
Mean & $21 \pm 2$ & $31 \pm 2$ & $<0.001$ \\
\hline
\end{tabular}

$L C X$ left circumflex coronary artery, $L A D$ left anterior descending coronary artery, $R C A$ right coronary artery, TIMI thrombolysis in myocardial infarction

and percentages, and continuous variables were expressed as the mean and standard deviation. Continuous variables with a normal distribution were compared using Student's t-test. Kruskal-Wallis test and one-way ANOVA were used to analyze the differences between groups. The categorical variables were tested by chi-square test. Spearman $\rho$ test correlation analysis was used to examine the correlation between Lp-PLA $\mathrm{A}_{2}$ and other risk factors. We used multivariate logistic regression analysis to assess the value of Lp-PLA ${ }_{2}$ in predicting the presence of CSF with adjustment for risk factors. Additionally, a receiver operating characteristic (ROC) curve was plotted for the plasma LpPLA2 level to evaluate the ability of the variable to classify the severity of CSF. The ROC curve were calculated in an area under the curve (AUC) and 95\% confidence intervals. A $P$ value $<0.05$ (two tailed) was considered significant.

\section{Results}

\section{Patient characteristics}

The baseline clinical characteristics of the study participants are shown in Table 1 . We enrolled 170 patients (mean age $61.6 \pm 9.7,60.6 \%$ male) who underwent coronary angiography; the sample was composed of 78 patients (mean age 60.2 $\pm 9.7,70.5 \%$ male) with CSF and 92 patients (mean age $62.7 \pm 9.5,52.1 \%$ male) with normal coronary flow (NCF). As shown in Table 1, men were more prevalent in the CSF group than in the NCF group $(70.5 \%$ versus $52.1 \%, P=0.018)$. However, the other demographic factors, such as age, BMI, waist circumference, prevalence of hypertension, prevalence of diabetes and current smoking, did not differ significantly between the CSF and NCF groups. Except for TGs, TC, and high-density lipoprotein cholesterol (HDL-c), the two groups showed no significant differences in most laboratory tests. The levels of HDL-C and TC were lower in the CSF group than in the NCF group $(1.15 \pm 0.07$ versus $1.49 \pm 0.29, \mathrm{P}<0.001 ; 4.32 \pm 0.85$ versus $4.76 \pm$ 1.01, $P=0.003)$, and the TG level was significantly higher in the CSF group $(1.97 \pm 1.57$ versus $1.51 \pm 0.66$, $P=0.020)$.

Lp-PLA 2 levels in the CSF group were significantly higher than those in the control group (288.6 \pm 50.3 versus $141.9 \pm 49.7, P<0.001$ ) (Table 1,Fig. 1 ). The TFC values of the two groups of coronary arteries were calculated respectively. The mean TFCs of LAD, LCX and RCA were significantly higher in CSF patients than in NCF patients $(p<0.001$ for each coronary artery, Table 2 , Fig. 2).

The spearman analysis, shown in Table 3, showed that the plasma Lp-PLA2 level was positively correlated with mean TFC $(\mathrm{r}=0.790, \mathrm{P}<0.001)$ and $\mathrm{C}$-reactive protein (CRP), $(\mathrm{r}=0.179, P=0.019)$, and negatively correlated with HDL-C $(\mathrm{r}=-0.693, \mathrm{P}<0.001)$ and ejection fraction ( $\mathrm{r}=-0.164, P=0.033$ ) (Figs. 3,4$)$. Univariate and multivariate logistic regression analyses were used to explore the associations of risk factors with CSF. The results indicated that the level of Lp-PLA $2(\mathrm{OR}=1.049, \mathrm{CI}=$ 1.034-1.064, $P<0.001)$, male sex $(\mathrm{OR}=2.192, \quad \mathrm{CI}=$ 1.161-4.140, $P=0.016)$ and hypertension $(\mathrm{OR}=1.965$, $\mathrm{CI}=1.034-3.736, P=0.039)$ were risk factors for CSF.

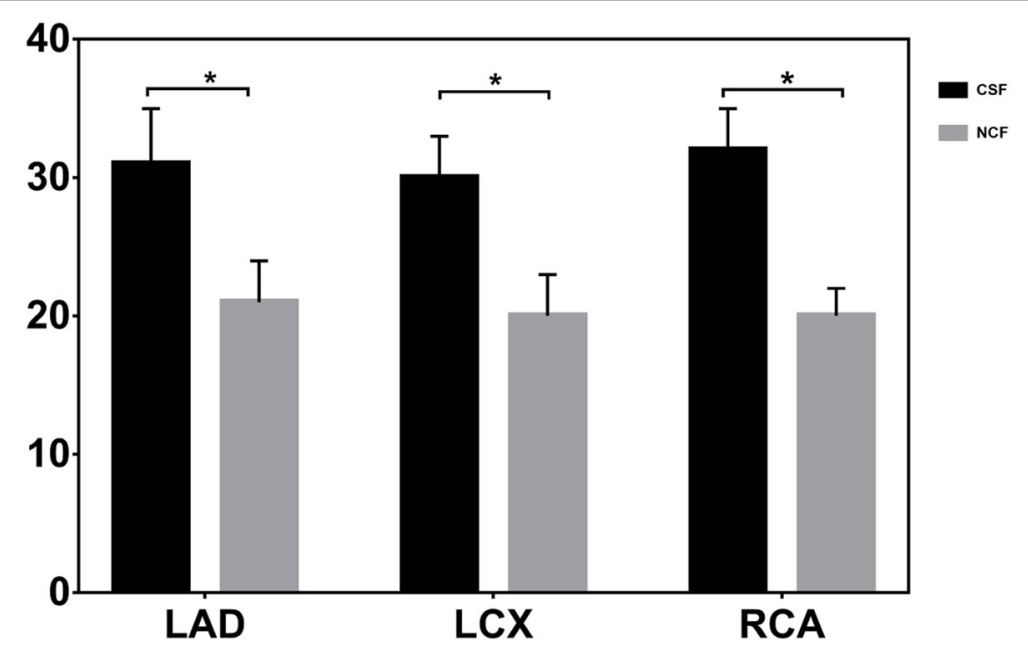

Fig. 2 Angiographic bar graph for Subjects With Slow Flow and normal flow. LAD left anterior descending coronary artery, LCX left circumflex coronary artery, RCA right coronary artery, CSF coronary slow flow, NSF normal slow flow 
Table 3 Spearman's correlation coefficients of Lp-PLA2 level with covariates

\begin{tabular}{lll}
\hline Variable & Correlation coefficient & $P$ value \\
\hline Age & -0.094 & 0.223 \\
BMI & 0.038 & 0.625 \\
Waistline & 0.087 & 0.261 \\
FBG & 0.122 & 0.114 \\
HbA1C & -0.028 & 0.722 \\
TG & 0.277 & $<0.001$ \\
TC & -0.198 & 0.010 \\
HDL-C & -0.693 & $<0.001$ \\
LDL-C & 0.061 & 0.432 \\
Creatinine & 0.088 & 0.255 \\
Hs-CRP & 0.179 & 0.019 \\
WBC & 0.084 & 0.274 \\
Ejection fraction & -0.164 & 0.033 \\
TFC & 0.790 & $<0.001$ \\
\hline
\end{tabular}

$B M I$ Body mass index, FBG Fasting blood glucose, HbA1c Glycosylated hemoglobin A1c, LDL-c Low density lipoprotein cholesterol, HDL-c High density lipoprotein cholesterol, TC total cholesterol, TG triglyceride, Hs-CRP Hyper-sensitive C-reactive protein, WBC white blood cell

After adjustment for traditional confounders, we found that the Lp-PLA ${ }_{2}$ levels remained to be significantly and independently associated with the presence of CSF $(\mathrm{OR}=1.040$, $\mathrm{CI}=1.022-1.059, P<0.001)$ (Table 4, Table 5, Fig. 5).

The ROC curve analysis (Fig. 6) showed that Lp-PLA 2 (AUC values $=0.978 ; C I=0.959-0.993 ; P<0.0001)$ was better in predicting CFS than TC $(0.621 ; \mathrm{CI}=0.537-$ $0.706 ; P=0.0064)$, TG $(0.603 ; \quad \mathrm{CI}=0.518-0.688 ; P=$ $0.021)$, male $\operatorname{sex}(0.592 ; \mathrm{CI}=0.506-0.677 ; P=0.040)$ and hypertension $(0.576 ; \mathrm{CI}=0.503-0.645 ; P=0.036)$. The sum of the sensitivity and specificity for the prediction

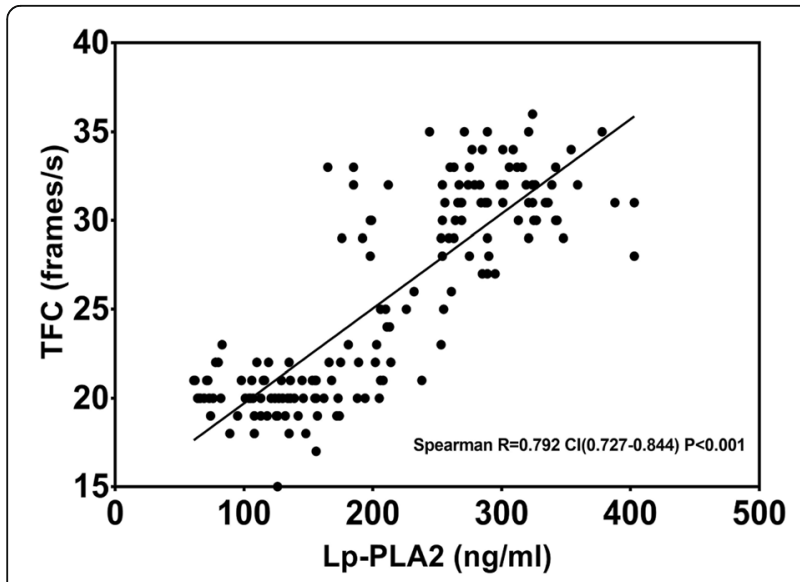

Fig. 3 Spearman correlation analysis of $L p-P L A_{2}$ value with coronary artery TIMI frame count. $L p-P L A_{2}$ levels were significantly positively correlated with TFC. Lp-PLA 2 lipoprotein-associated phospholipase A2, TFC thrombolysis in myocardial infarction frame count

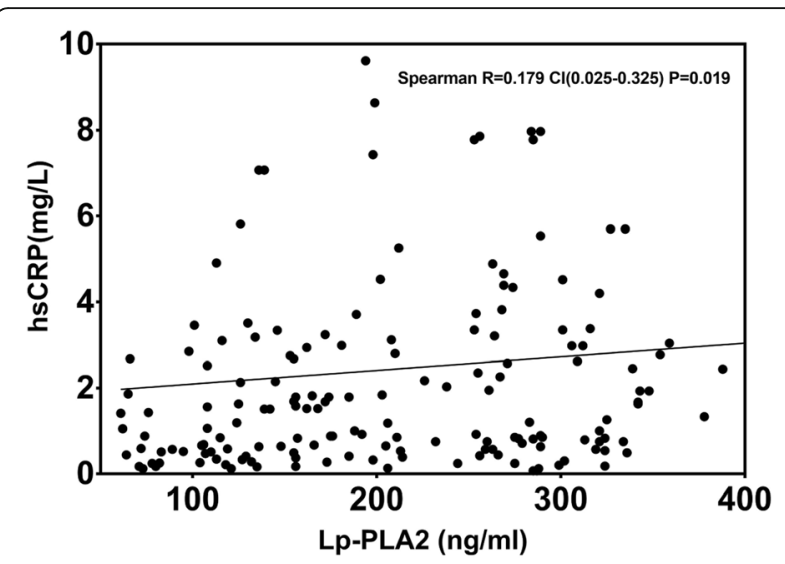

Fig. 4 Spearman correlation analysis of $L p-P L A_{2}$ value with hs-CRP, $L p-P L A_{2}$ levels were significantly positively correlated with hs-CRP. Lp-PLA 2 lipoprotein-associated phospholipase A2, hs-CRP

of the extent of CSF was maximal at a level of LpPLA2 $\geq 260.5 \mathrm{ng} / \mathrm{ml}$ (sensitivity $=76.9 \%$ [95\% CI 66 to $85.71 \%]$, specificity $=98.9 \%$ [ $95 \%$ CI 94.1 to $99 \%]$ ).

\section{Discussion}

As far as we know, the present study demonstrated for the first time that there was an independent relationship between Lp-PLA ${ }_{2}$ and CSF phenomenon. The major

Table 4 Univariate regression analysis of the association of coronary slow flow with variables

\begin{tabular}{|c|c|c|c|}
\hline Variables & OR & $95 \% \mathrm{Cl}$ & $P$ value \\
\hline Age & 0.972 & $0.942-1.004$ & 0.087 \\
\hline Male & 2.192 & $1.161-4.140$ & 0.016 \\
\hline $\mathrm{BMI}$ & 1.055 & $0.945-1.177$ & 0.339 \\
\hline Waistline & 1.019 & $0.989-1.050$ & 0.216 \\
\hline Diabetes mellitus & 1.153 & $0.556-2.391$ & 0.703 \\
\hline Hypertension & 1.965 & $1.034-3.736$ & 0.039 \\
\hline Smoking & 1.119 & $0.461-2.714$ & 0.804 \\
\hline $\mathrm{FBG}$ & 1.071 & $0.911-1.258$ & 0.407 \\
\hline $\mathrm{HbA} 1 \mathrm{c}$ & 0.953 & $0.871-1.042$ & 0.288 \\
\hline $\mathrm{TC}$ & 0.603 & $0.427-0.850$ & 0.004 \\
\hline TG & 1.618 & $1.071-2.443$ & 0.022 \\
\hline LDL & 0.682 & $0.454-1.025$ & 0.066 \\
\hline $\mathrm{HDL}$ & 0.991 & $0.955-1.06$ & 0.244 \\
\hline Creatinine & 1.001 & $0.989-1.014$ & 0.869 \\
\hline $\mathrm{Hs}-\mathrm{CRP}$ & 1.081 & $0.975-1.197$ & 0.138 \\
\hline WBC & 1.107 & $0.918-1.336$ & 0.287 \\
\hline Ejection fraction & 0.961 & $0.916-1.008$ & 0.104 \\
\hline Lp-PLA 2 & 1.049 & $1.034-1.064$ & $<0.001$ \\
\hline
\end{tabular}

$B M I$ Body mass index, FBG Fasting blood glucose, HbA1c Glycosylated hemoglobin A1c, LDL-c Low density lipoprotein cholesterol, HDL-c High density lipoprotein cholesterol, TC total cholesterol, TG triglyceride, $H s-C R P$ hyper-sensitive C-reactive protein, WBC white blood cell 
Table 5 Multivariate logistic regression analysis of the risk of CSF with Lp-PLA 2

\begin{tabular}{llll}
\hline & OR & $95 \% \mathrm{Cl}$ & $\mathrm{P}$ \\
\hline Model 1 & 1.031 & $1.022-1.040$ & $<0.001$ \\
Model 2 & 1.041 & $1.022-1.061$ & $<0.001$ \\
Model 3 & 1.040 & $1.022-1.059$ & $<0.001$ \\
\hline
\end{tabular}

Odds ratio and $95 \%$ confidence intervals $(\mathrm{Cl})$ were obtained by the multivariate logistic regression model: model 1:After adjustment the age, gender, BMI, hypertension, Diabetes mellitus, smoking; model 2: model 1+ laboratory parameters in Fig. 1; model 3: model 2 + medicine

finding was that Lp-PLA 2 levels were positively correlated with CSF and significantly elevated in subjects with CSF. In addition, multivariate logistic regression analysis and ROC curves showed that Lp-PLA 2 was still independently associated with the presence and severity of CSF. Male sex and hypertension also independently predicted the presence of the CSF phenomenon.

Although CSF has been found to be related to microvascular diseases, vascular endothelial dysfunction, coronary atherosclerosis, oxidative stress, insulin resistance, adipocytokines, and abnormal blood composition, the exact mechanisms are still unknown [18-20]. Tambe proposed that the CSF phenomenon might be related to abnormal microcirculation in 1972 [2]. Oğuzhan Çelik et al. identified a correlation between the extent of disruption in endothelial function and the CSF level using the flowmediated dilation (FMD) method [21]. Several studies have found that the imbalance between endothelin-1 and nitric oxide in patients with CSF supports the involvement of endothelial dysfunction in CSF etiopathogenesis [22]. An intravascular ultrasound study demonstrated that diffuse coronary calcification was present in $88 \%$ of CSF patients, and statin therapy has been shown to improve coronary flow in CSF subjects [23]. Decreased fractional flow reserve in CSF patients has been demonstrated by intravascular ultrasound (IVUS) to result in increased resistance in the epicardial coronary artery due to diffuse atherosclerotic disease [24].

The role of chronic inflammation-mediated endothelial injury in CSF has been widely recognized. Muhammed Oylumlu et al. revealed that the platelet-to-lymphocyte ratio could be an important risk factor in patients with CSF [25]. Li et al. showed that plasma CRP and IL-6 as inflammatory markers were positively correlated with TIMI frame count in patients with CSF [26]. In our study, Lp$\mathrm{PLA}_{2}$ was shown to be an independent predictor of CSF. Lp-PLA $A_{2}$, known as platelet-activating factor acetylhydrolase, is a calcium-independent serine lipase [10]. Lp-PLA is synthesized by macrophages and other inflammatory cells and circulates in the human blood mainly with LDL granules (80-85\%) and less often with HDL [9]. Lp-PLA 2 is involved in the oxidative modification of vascular wall LDL to generate oxidized phospholipids and oxidized non-esterified fatty acids, which can promote the development of vascular inflammation and atherosclerotic plaques [27]. Lp-PLA $A_{2}$ has been identified as an independent predictor of cardiovascular disease (CVD), and has been recommended as an adjunct to traditional risk assessment in patients with moderate and high 10-year risk of CVD as defined by Framingham risk scores [28]. In the REGARDS study, Lp-PLA ${ }_{2}$ activity was associated with coronary heart disease (CHD) risk over 5.3 years based on Cox proportional hazards regression [29]. Our study found that in the CSF group, with the increase in TFC, Lp-PLA2 levels exhibited an increasing trend, and there was a significant positive correlation between CSF and Lp-PLA 2 . Additionally multivariate logistic regression analysis showed that Lp-PLA $A_{2}$ could independently predict CSF. However, the mechanisms underlying the relationship between Lp-PLA 2

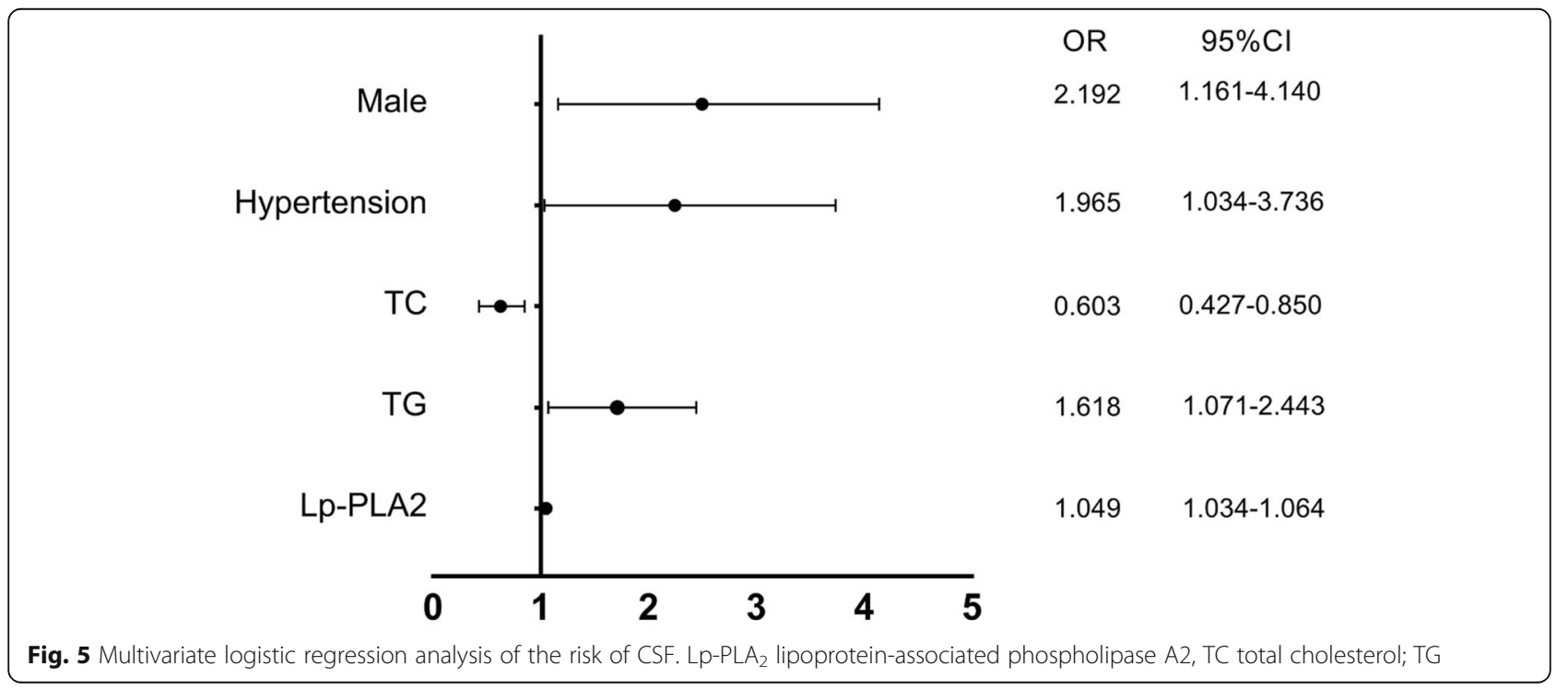



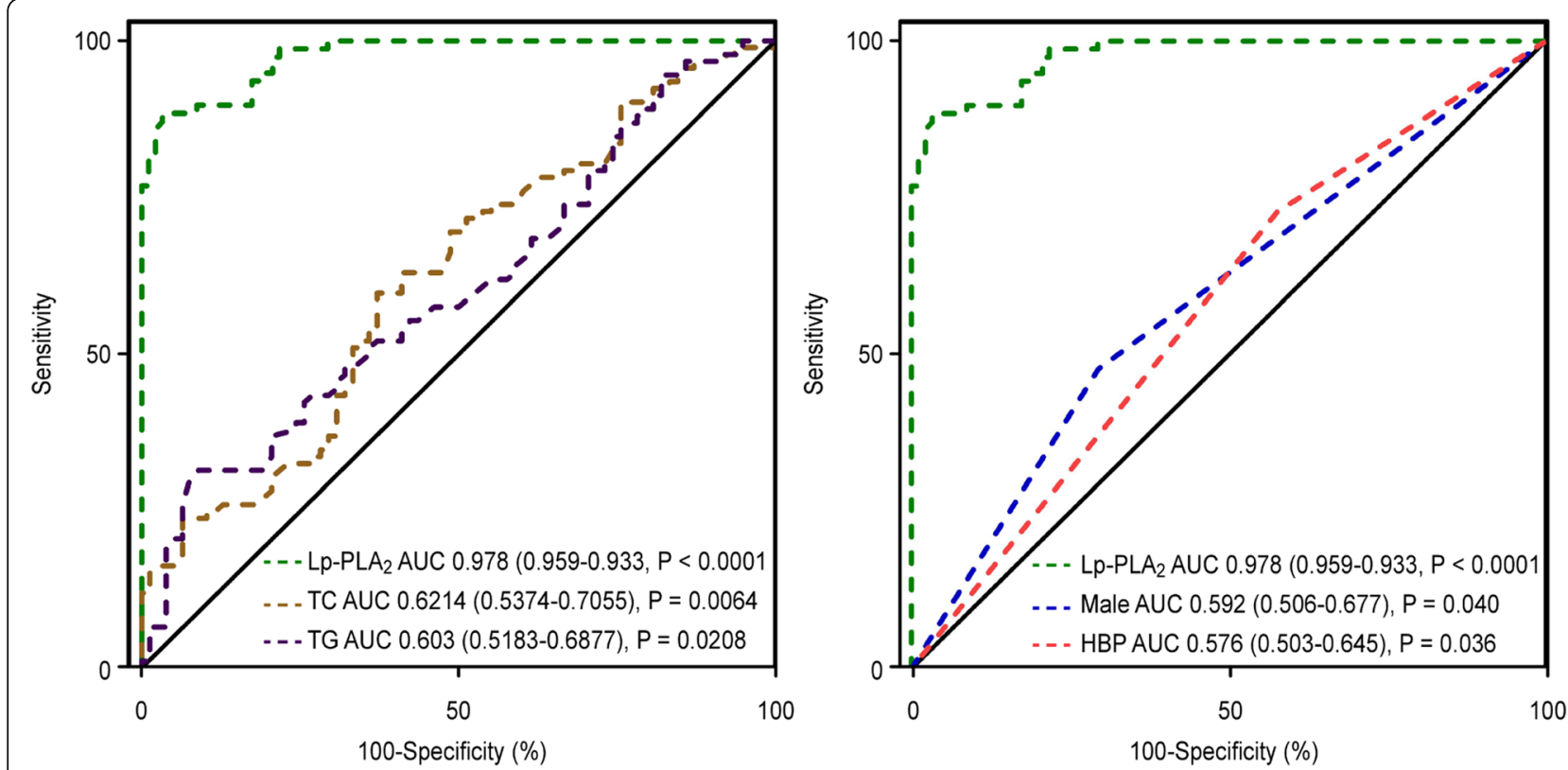

Fig. 6 Receiver-operating characteristic curve analysis showing the prognostic value of $L p-P L A_{2}, T C, T G s$, male sex, and hypertension. $L p-P L A_{2}$ lipoprotein-associated phospholipase A2, TC total cholesterol; TG triglyceride, HBP hypertension

and CSF are not entirely understood. The most reliable hypothesis seems to be that CSF is caused by increased microvascular resistance induced by endothelial dysfunction due to Lp-PLA 2 mediated chronic inflammation.

Numerous studies have attempted to define the demographics, characteristics and independent predictors of patients with CSF. Arbey Y found that current smoking was associated with CSF [30]. Other studies have shown that BMI and hypertension were predictors of CSF phenomena [31]. Compared to other studies, our study did not find an association between BMI and CSF. Our study also shows that Lp-PLA, male gender,c and hypertension are independent risk factor of the presence of CSF phenomena. Tsimikas found in his study that there was a significant correlation between Lp-PLA ${ }_{2}$ and LDL, non-HDL, and HDL [11]. However, we did not find any difference in LDL between the two groups, which may be related to the use of lipid-lowering drugs.

\section{Limitations}

Our study has some limitations: (1)This study was limited by a small and nonrandomized sample, which may restrict the generalizability of our results. (2)The study did not examine the ethnic background or socioeconomic status of the study population.(3)This study was also a single center, retrospective study. Multicenter randomized controlled trials must be conducted to explore the relationship between Lp-PLA 2 and CSF. (4)The use of drugs, such as lipid-lowering drugs, may affect the results of experiments. (5)Finally, we determined only the mass of Lp-PLA ${ }_{2}$ instead of its activity due to our laboratory limitations.

\section{Conclusion}

In summary, the main finding was that Lp-PLA 2 levels were positively correlated with CSF. The multivariate regression results showed that Lp-PLA ${ }_{2}$ was independently associated with the presence and severity of CSF. Currently, there is still no effective method to treat CSF. This study indicated the association between Lp-PLA and CSF, which may provide a new therapeutic target for the treatment of CSF. However, the exact pathophysiological mechanisms of Lp-PLA 2 in CSF require further study to elucidate.

\section{Abbreviations}

CSF: Coronary slow flow; Lp-PLA 2 : Lipoprotein-associated phospholipase A2; TFC: Thrombolysis in myocardial infarction frame count; RCA: Right coronary artery; LCX: Left circumflex coronary artery; LAD: Left anterior descending coronary artery; CVD: Cardiovascular disease; TC: Total cholesterol; TG: Triglyceride; HDL-C: High-density lipoprotein cholesterol; LDL-C: Lowdensity lipoprotein cholesterol; HbA1c: Glycosylated hemoglobin A1c; FBG: Fasting blood glucose; BMI: Body mass index; CRP: C-reactive protein; ROC: Receiver-operating characteristic curve

\section{Acknowledgements}

Not applicable.

\section{Authors' contributions}

$Y$-DD carried out the experiments, acquired the data and wrote the first draft of the paper; Y-QP carried out the experiments and wrote sections of the manuscript; Y-XZ, X-LL, HS and QM recruited the subjects, performed the patients assessments and critically reviewed the paper for intellectual content. Y-DD, Y-QP, R-W, J-XY and SZ performed the statistical analyses; $\mathrm{H}-$ LG conceived and designed the study and handled funding and supervision. All authors read and approved the final manuscript. 


\section{Funding}

This work was supported by grants 81573744,81973841 from the China National Natural Scientific Foundation.

\section{Availability of data and materials}

The data and materials can be used with permission.

\section{Ethics approval and consent to participate}

The study was approved by the Beijing Anzhen Hospital Ethics Committee of Capital Medical University, and all participants signed an informed consent form.

\section{Consent for publication}

All participants provided written informed consent before enrollment in this study.

\section{Competing interests}

The authors declare that they have no competing interests.

\section{Author details}

'Department of Cardiology, Beijing Anzhen Hospital, Capital Medical University, Beijing 100029, PR China. ${ }^{2}$ Beijing Tiantan Hospital, Capital Medical University, Beijing, China.

Received: 20 February 2020 Accepted: 2 April 2020

Published online: 27 May 2020

\section{References}

1. Horjeti B, Goda A. Acute ischemia manifestation in a patient with coronary slow flow phenomenon. J Electrocardiol. 2012;45(3):277-9.

2. Tambe AA, Demany MA, Zimmerman HA, Mascarenhas E. Angina pectoris and slow flow velocity of dye in coronary arteries--a new angiographic finding. Am Heart J. 1972;84(1):66-71.

3. Jin Z, Tan Q, Sun B. Telmisartan ameliorates vascular endothelial dysfunction in coronary slow flow phenomenon (CSFP). Cell Biochem Funct. 2018;36(1):18-26.

4. Yilmaz M, Korkmaz H, Bilen MN, Uku O, Kurtoglu E. Could neutrophil/ lymphocyte ratio be an indicator of coronary artery disease, coronary artery ectasia and coronary slow flow? J Int Med Res. 2016;44(6):1443-53.

5. Hawkins BM, Stavrakis S, Rousan TA, Abu-Fadel M, Schechter E. Coronary slow flow--prevalence and clinical correlations. Circ J. 2012;76(4):936-42.

6. Yayla C, Akboga MK, Gayretli Yayla K, Ertem AG, Efe TH, Sen F, Unal S, Acar $B$, Ozcan $F$, Turak $O$, et al. A novel marker of inflammation in patients with slow coronary flow: lymphocyte-to-monocyte ratio. Biomark Med. 2016; 10(5):485-93.

7. Mutluer FO, Ural D, Gungor B, Bolca O, Aksu T. Association of Interleukin-1 gene cluster polymorphisms with coronary slow flow phenomenon. Anatolian J Cardiol. 2018;19(1):34-41.

8. Shi GL, Cai XX, Su YM, Chen C, Deng XT, Pan HY, Fan MK, Zhu JH, Pan M. Interleukin-10 promotor -592A/C polymorphism is associated with slow coronary flow in Han Chinese. Int J Clin Exp Pathol. 2015;8(4):4091-8.

9. Silva IT, Mello AP, Damasceno NR. Antioxidant and inflammatory aspects of lipoprotein-associated phospholipase a(2) (Lp-PLA(2)): a review. Lipids Health Dis. 2011;10:170.

10. Stafforini DM. Plasma PAF-AH (PLA2G7): biochemical properties, association with LDLs and HDLs, and regulation of expression. Enzymes. 2015;38:71-93.

11. Acevedo M, Varleta P, Kramer V, Valentino G, Quiroga T, Prieto C, Parada J, Adasme M, Briones L, Navarrete C. Comparison of lipoprotein-associated phospholipase A2 and high sensitive C-reactive protein as determinants of metabolic syndrome in subjects without coronary heart disease: in search of the best predictor. Int J Endocrinol. 2015;2015:934681.

12. Ballantyne CM, Hoogeveen RC, Bang H, Coresh J, Folsom AR, Heiss G, Sharrett AR. Lipoprotein-associated phospholipase A2, high-sensitivity Creactive protein, and risk for incident coronary heart disease in middle-aged men and women in the atherosclerosis risk in communities (ARIC) study. Circulation. 2004;109(7):837-42.

13. Roffi M, Patrono C, Collet JP, Mueller C, Valgimigli M, Andreotti F, Bax JJ, Borger MA, Brotons C, Chew DP, et al. 2015 ESC guidelines for the management of acute coronary syndromes in patients presenting without persistent ST-segment elevation: task force for the Management of Acute Coronary Syndromes in patients presenting without persistent ST-segment elevation of the European Society of Cardiology (ESC). Eur Heart J. 2016; 37(3):267-315.

14. Richardson P, McKenna W, Bristow M, Maisch B, Mautner B, O'Connell J, Olsen E, Thiene G, Goodwin J, Gyarfas I, et al. Report of the 1995 World Health Organization/international society and Federation of Cardiology Task Force on the definition and classification of cardiomyopathies. Circulation. 1996;93(5):841-2.

15. Alberti KG, Zimmet PZ. Definition, diagnosis and classification of diabetes mellitus and its complications. Part 1: diagnosis and classification of diabetes mellitus provisional report of a WHO consultation. Diab Med. 1998;15(7):539-53.

16. James PA, Oparil S, Carter BL, Cushman WC, Dennison-Himmelfarb C, Handler J, Lackland DT, LeFevre ML, MacKenzie TD, Ogedegbe O, et al. 2014 evidence-based guideline for the management of high blood pressure in adults: report from the panel members appointed to the eighth joint National Committee (JNC 8). Jama. 2014:311(5):507-20.

17. Gibson CM, Cannon CP, Daley WL, Dodge JT Jr, Alexander B Jr, Marble SJ, McCabe CH, Raymond L, Fortin T, Poole WK, et al. TIMl frame count: a quantitative method of assessing coronary artery flow. Circulation. 1996; 93(5):879-88.

18. Tasolar H, Eyyupkoca F, Akturk E, Karakus Y, Cansel M, Yagmur J, Ozyalin F, Altun B, Pekdemir $\mathrm{H}$. Endothelial nitric oxide synthase levels and their response to exercise in patients with slow coronary flow. Cardiovasc J Afr. 2013;24(9-10):355-9.

19. Akboga MK, Canpolat U, Balci KG, Akyel A, Sen F, Yayla C, Cay S, Aras D, Aydogdu S. Increased platelet to lymphocyte ratio is related to slow coronary flow. Angiology. 2016;67(1):21-6.

20. Demir B, Caglar IM, Tureli HO, Pirhan O, Aciksari G, Serkan C, Cem O, Gedikbasi A, Zorkun C, Demir E, et al. Coronary slow flow phenomenon associated with high serum levels of soluble CD40 ligand and urotensin II: a multi-marker approach. Clin Lab. 2014;60(11):1909-20.

21. Li Y, Zhang H, Liang Y, Wang W, Xu T, Zhang J, Xiao W, Wang T. Effects of hyperbaric oxygen on vascular endothelial function in patients with slow coronary flow. Cardiol J. 2018;25(1):106-12.

22. Pekdemir H, Polat G, Cin VG, Camsari A, Cicek D, Akkus MN, Doven O, Katircibasi MT, Muslu N. Elevated plasma endothelin-1 levels in coronary sinus during rapid right atrial pacing in patients with slow coronary flow. Int J Cardiol. 2004;97(1):35-41.

23. Camsari A, Ozcan T, Ozer C, Akcay B. Carotid artery intima-media thickness correlates with intravascular ultrasound parameters in patients with slow coronary flow. Atherosclerosis. 2008;200(2):310-4.

24. Pekdemir H, Cin VG, Cicek D, Camsari A, Akkus N, Doven O, Parmaksiz HT. Slow coronary flow may be a sign of diffuse atherosclerosis. Contribution of FFR and IVUS. Acta Cardiol. 2004;59(2):127-33.

25. Oylumlu M, Dogan A, Oylumlu M, Yildiz A, Yuksel M, Kayan F, Kilit C, Amasyali B. Relationship between platelet-to-lymphocyte ratio and coronary slow flow. Anatolian J Cardiol. 2015;15(5):391-5.

26. Li JJ, Qin XW, Li ZC, Zeng HS, Gao Z, Xu B, Zhang CY, Li J. Increased plasma C-reactive protein and interleukin- 6 concentrations in patients with slow coronary flow. Clin chim Acta. 2007;385(1-2):43-7.

27. Di Pietro N, Formoso G, Pandolfi A. Physiology and pathophysiology of oxLDL uptake by vascular wall cells in atherosclerosis. Vasc Pharmacol. 2016;84:1-7.

28. Davidson MH, Corson MA, Alberts MJ, Anderson JL, Gorelick PB, Jones PH, Lerman A, McConnell JP, Weintraub HS. Consensus panel recommendation for incorporating lipoprotein-associated phospholipase A2 testing into cardiovascular disease risk assessment guidelines. Am J Cardiol. 2008; 101(12a):51f-7f.

29. De Stefano A, Mannucci L, Massoud R, Bernardini S, Cortese C. Performance characteristics of lipoprotein-associated phospholipase A2 activity assay on the dimension vista analyser and preliminary study of a healthy Italian population. Biochem Med. 2017;27(3):030701.

30. Arbel Y, Rind E, Banai S, Halkin A, Berliner S, Herz I, Mashav N, Thurm T, Keren G, Finkelstein A. Prevalence and predictors of slow flow in angiographically normal coronary arteries. Clin Hemorheol Microcirc. 2012; 52(1):5-14.

31. Sanati H, Kiani R, Shakerian F, Firouzi A, Zahedmehr A, Peighambari M, Shokrian L, Ashrafi P. Coronary slow flow phenomenon clinical findings and predictors. Res Cardiovasc Med. 2016;5(1):e30296.

\section{Publisher's Note}

Springer Nature remains neutral with regard to jurisdictional claims in published maps and institutional affiliations. 